\title{
Rational design and self-assembly of coiled-coil linked SasG protein fibrils \\ (Supplementary information)
}

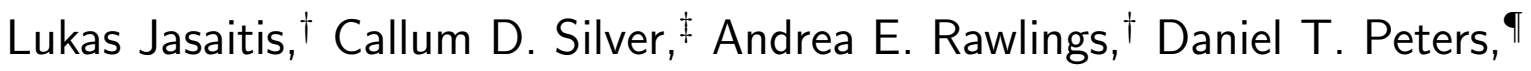
Fiona Whelan, ${ }^{\S}$ Lynne Regan," Laia Pasquina-Lemonche, ${ }^{\perp}$ Jennifer R. Potts,, ,@ Steven D. Johnson, ${ }^{\ddagger}$ and Sarah S. Staniland ${ }^{*, \dagger}$

$\dagger$ Department of Chemistry, University of Sheffield, Sheffield, S3 7HF, United Kingdom $\ddagger$ Department of Electronic Engineering, University of York, York, YO10 5DD, United Kingdom

\Biosciences Institute, Newcastle University, Newcastle upon Tyne, NE2 4HH, United Kingdom

$\S$ School of Biological Sciences, University of Adelaide, Adelaide, SA 5005, Australia || Institute for Quantitative Biology, Biochemistry and Biotechnology, University of Edinburgh, Edinburgh, EH9 3JU, Scotland

$\perp$ Department of Physics and Astronomy, University of Sheffield, Sheffield, S3 $7 H F$, United Kingdom

\#School of Life and Environmental Science, University of Sydney, Sydney, NSW 2006, Australia

@Department of Biology, University of York, York, YO10 5DD, United Kingdom

E-mail: S.S.Staniland@sheffield.ac.uk

Phone: +44 1142229539 


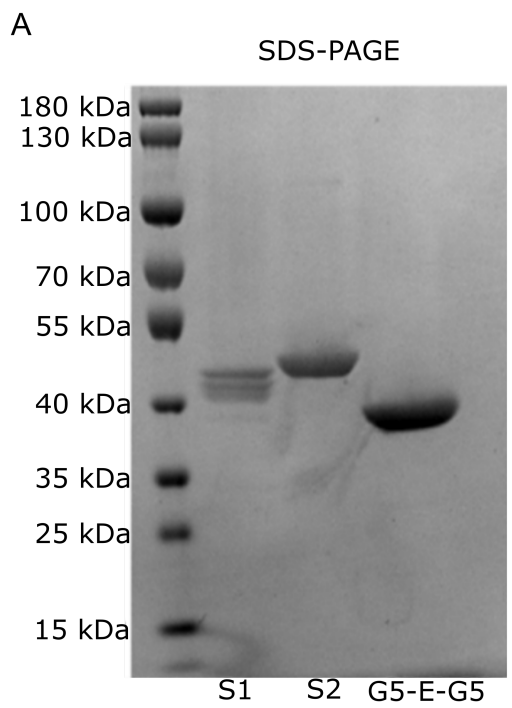

B
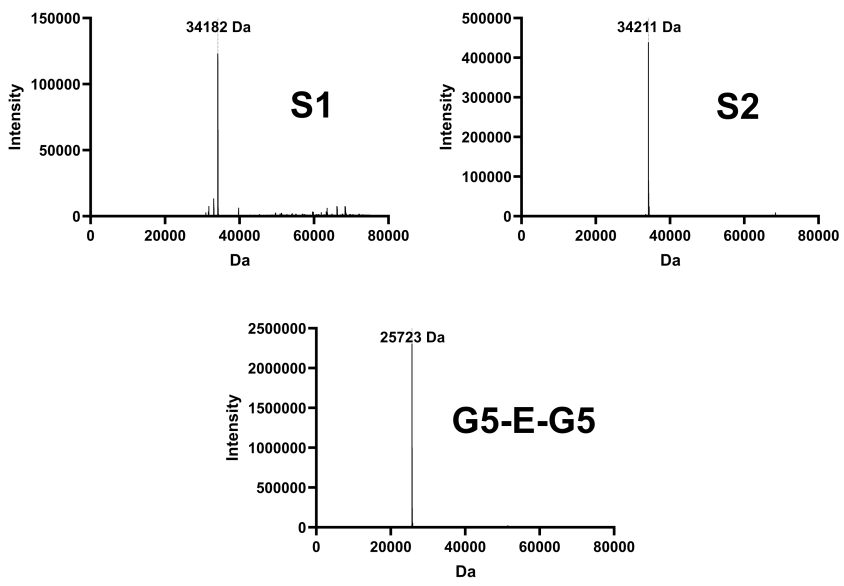

Supplementary Figure 1: Characterization of purified subunit masses. (A) Sodium Dodecyl Sulphate Polyacrylamide Gel Electrophoresis (SDS-PAGE) analysis of gel filtration purified subunits S1, S2 and G5-E-G5. The subunits run at apparent sizes above $40 \mathrm{kDa}$ (as opposed to theoretical mass of $34 \mathrm{kDa}$ ). There is a laddering effect observed in S1 lane. The same samples visualized by SDS-PAGE were analysed by electrospray ionization - time of flight (ESI-TOF) mass spectrometry, where correct protein sizes were confirmed (B), with no other species detected at significant levels. The measured masses match expected theoretical masses based on primary amino acid sequence: S1 (theoretical: 34181.35 Da; MS-peak: $34181.67 \mathrm{Da}$ ), S2 (theoretical: 34210.92 Da; MS-peak: $34211.00 \mathrm{Da})$ and G5-E-G5 (theoretical: 25722.64 Da; MS-peak: $25723 \mathrm{Da})$. 


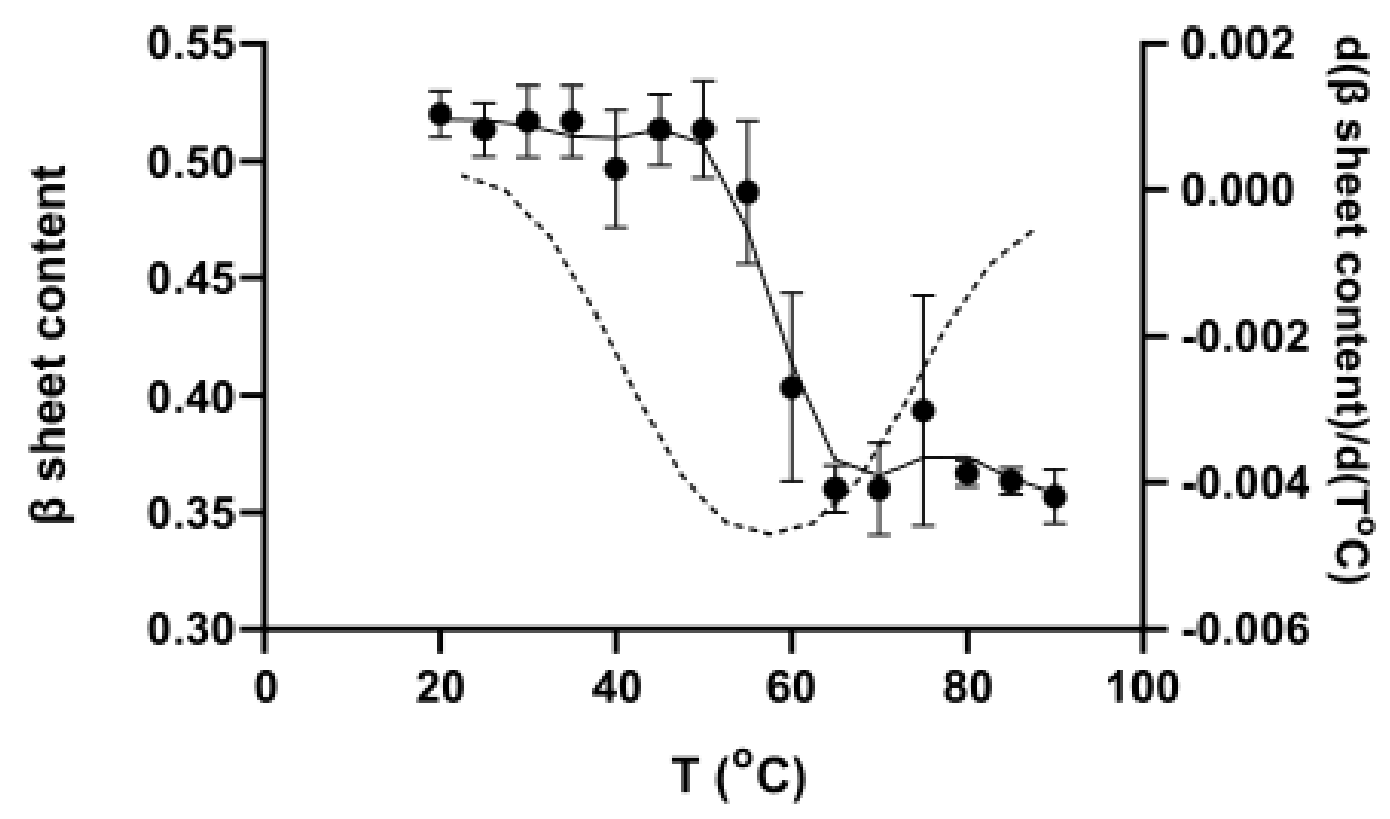

Supplementary Figure 2: Characterization of G5-E-G5 thermal stability Solid line: Betasheet fractions extracted from CD spectra at a range of temperature points using CDSSTR fitting algorithm (reference set SP180). Error bars are standard deviations (population) of three sets of thermal denaturation runs. The dashed line is the first order derivative of the curve (fitted to the dots). A $56.6{ }^{\circ} \mathrm{C}\left(\mathrm{STDEV}=0.58{ }^{\circ} \mathrm{C}\right)$ transition temperature can be inferred. 

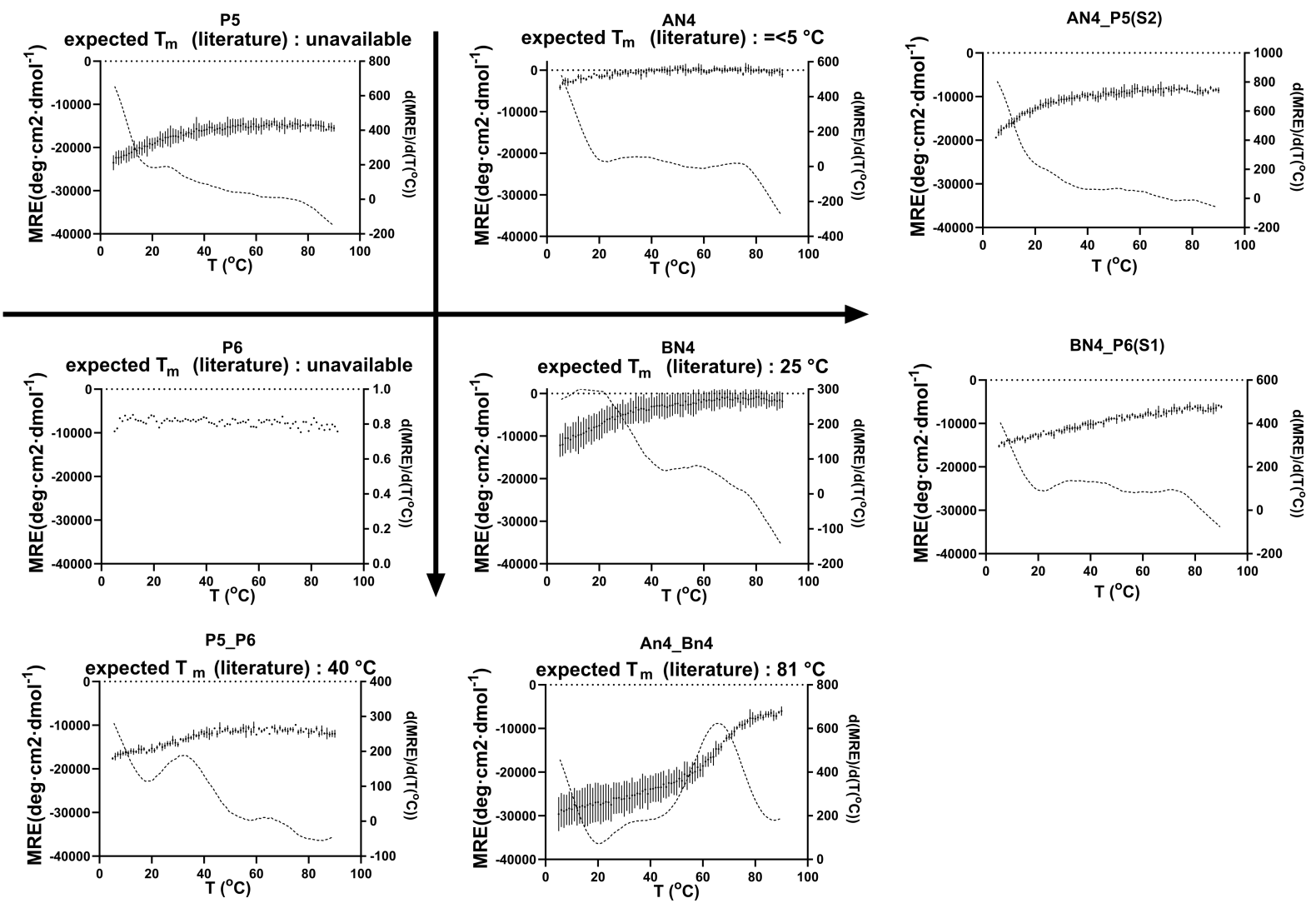

Supplementary Figure 3: CD thermal denaturation curves of individual alpha helices making up coiled coil pairs P5/P6 and An4/Bn4. Alpha helices P5, P6, AN4 and BN4 present in subunits $\mathrm{S} 1$ and $\mathrm{S} 2$ were cloned and purified to assess their melting temperatures individually, and in combination. Mixtures of P5 with P6 and An4 with Bn4 were assessed and compared to their values in the literature. $31^{\circ} \mathrm{C}\left(\mathrm{STDEV}=1.1{ }^{\circ} \mathrm{C}\right)$ and $66{ }^{\circ} \mathrm{C}\left(\mathrm{STDEV}=0.57{ }^{\circ} \mathrm{C}\right)$ were close to literature values of $40^{\circ} \mathrm{C}$ and $81^{\circ} \mathrm{C}$ for $\mathrm{P} 5 / \mathrm{P} 6$ and $\mathrm{An} 4 / \mathrm{Bn} 4$ respectively (the discrepancy may reflect different sample purities or temperature detection instrumentation used in the melting experiments). Looking at profiles of An4/P5 (alpha helices found in subunit S1) and Bn4/P6 (alpha helices found in subunit S1) there seems to be no clear interaction (as inferred from the absence of a significant shift towards higher transition temperatures). Error bars correspond to standard deviations (population) of each data point collected in triplicate. Dashed lines represent first order derivatives used to determine the transition temperatures. MRE - mean residue ellipticity. All variable temperature readings done at $222 \mathrm{~nm}$. 
$\mathrm{S} 1 \quad \mathrm{~S} 2 \quad \mathrm{~S} 1 \mathrm{~S} 2$
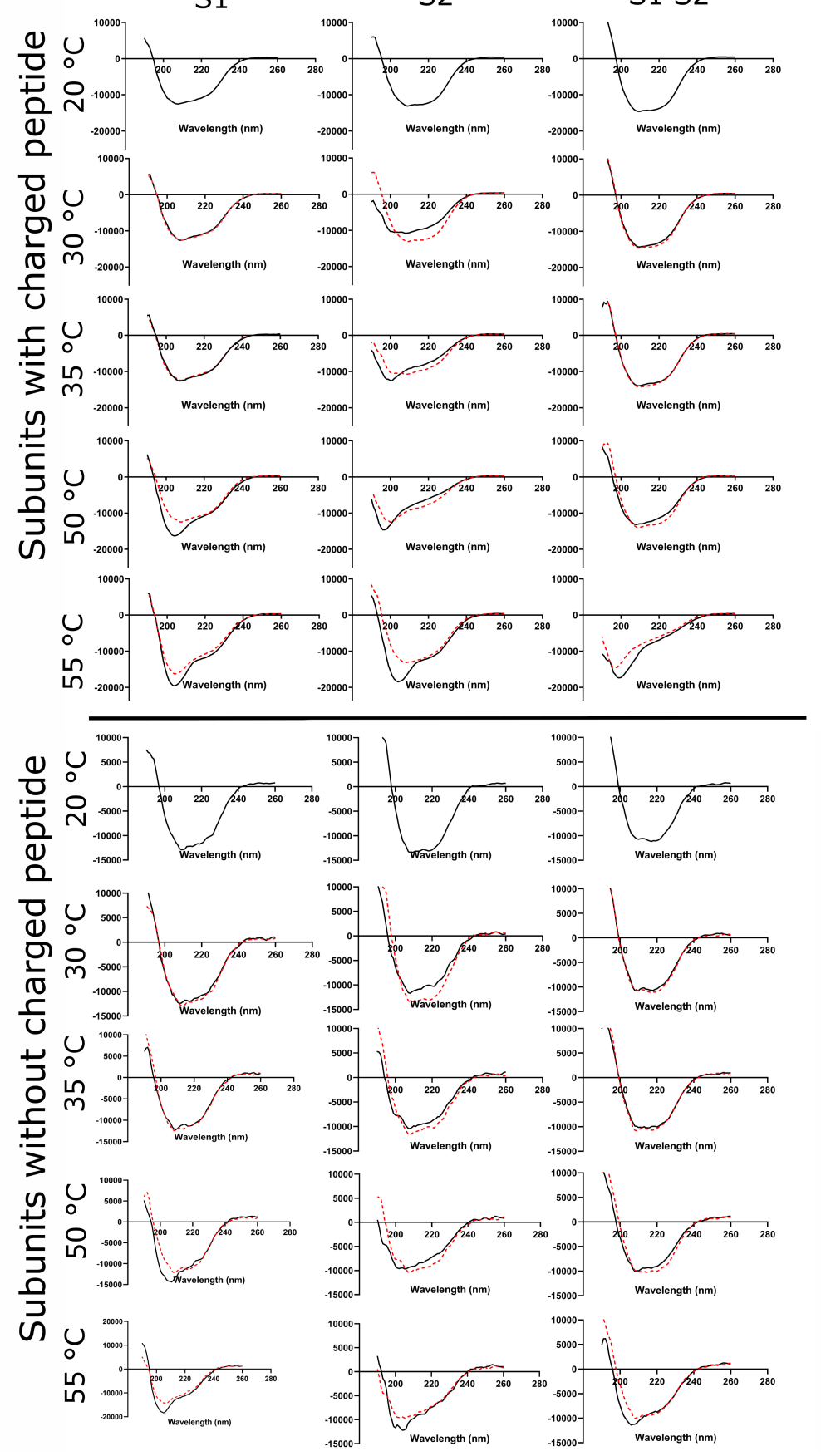

Supplementary Figure 4: Circular dichroism (CD) spectra of S1, S2 and S1/S2 at different temperatures (black line). The red puncture lines show spectra of a previous temperature point. The set at the top compares $\mathrm{S} 1, \mathrm{~S} 2$ and $\mathrm{S} 1 / \mathrm{S} 2$ with the C-terminal charged peptide at $20{ }^{\circ} \mathrm{C}, 30{ }^{\circ} \mathrm{C}, 35{ }^{\circ} \mathrm{C}, 50{ }^{\circ} \mathrm{C}$ and $55^{\circ} \mathrm{C}$. The bottom set compares S1, S2 and S1/S2 without the $\mathrm{C}$-terminal charged peptide at the same temperature points. In both cases, it can be seen that S1 retains a consistent spectrum until $35^{\circ} \mathrm{C}$, while $\mathrm{S} 2$ undergoes structural changes starting at $30{ }^{\circ} \mathrm{C}$. S1/S2 mixture does not change until the samples reach $55{ }^{\circ} \mathrm{C}$ in both sets of data. This shows that regardless of the presence of the peptide the subunits interact and the thermal properties remain the same. All Y-axis units are mean residue ellipticity. 


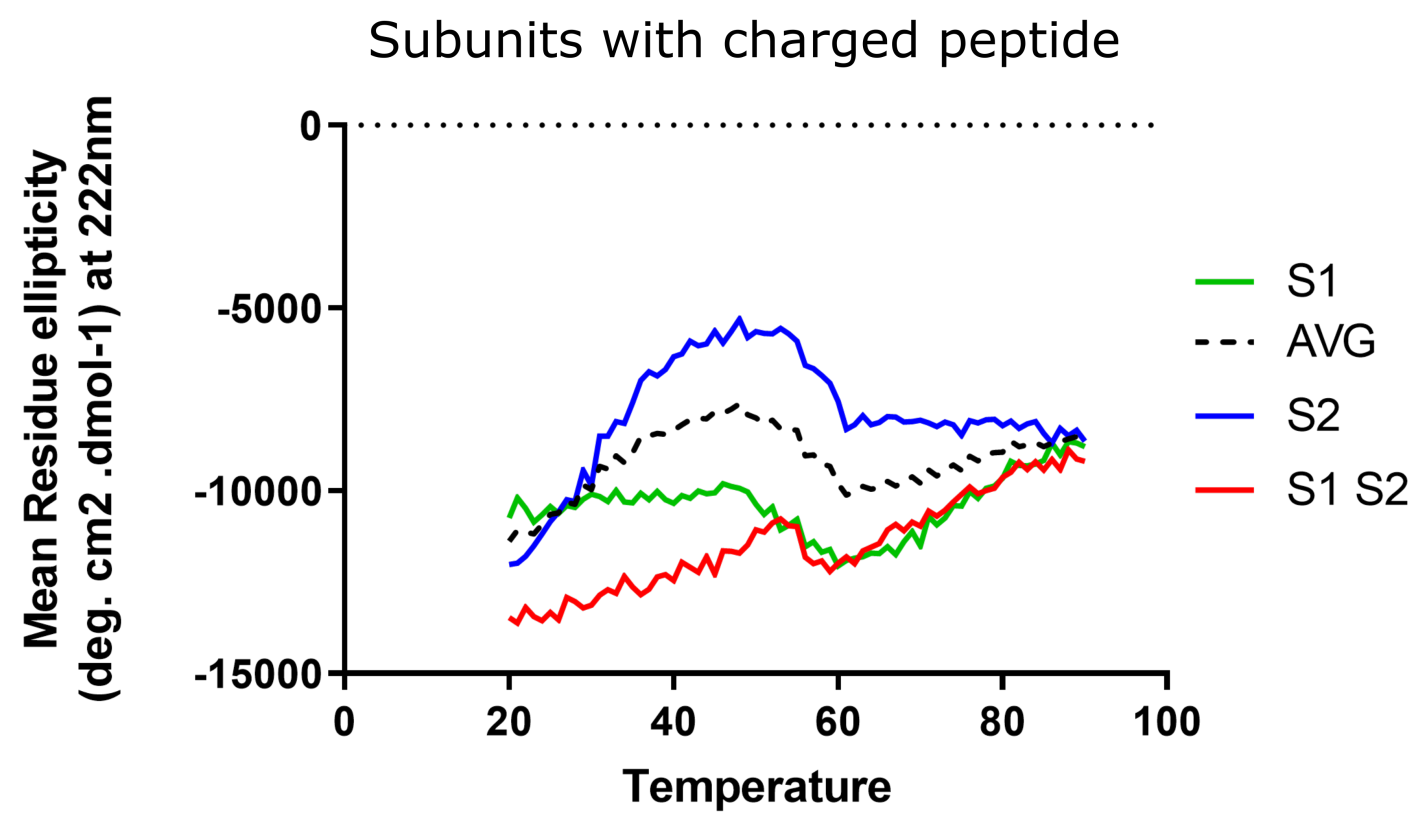

Subunits without charged peptide

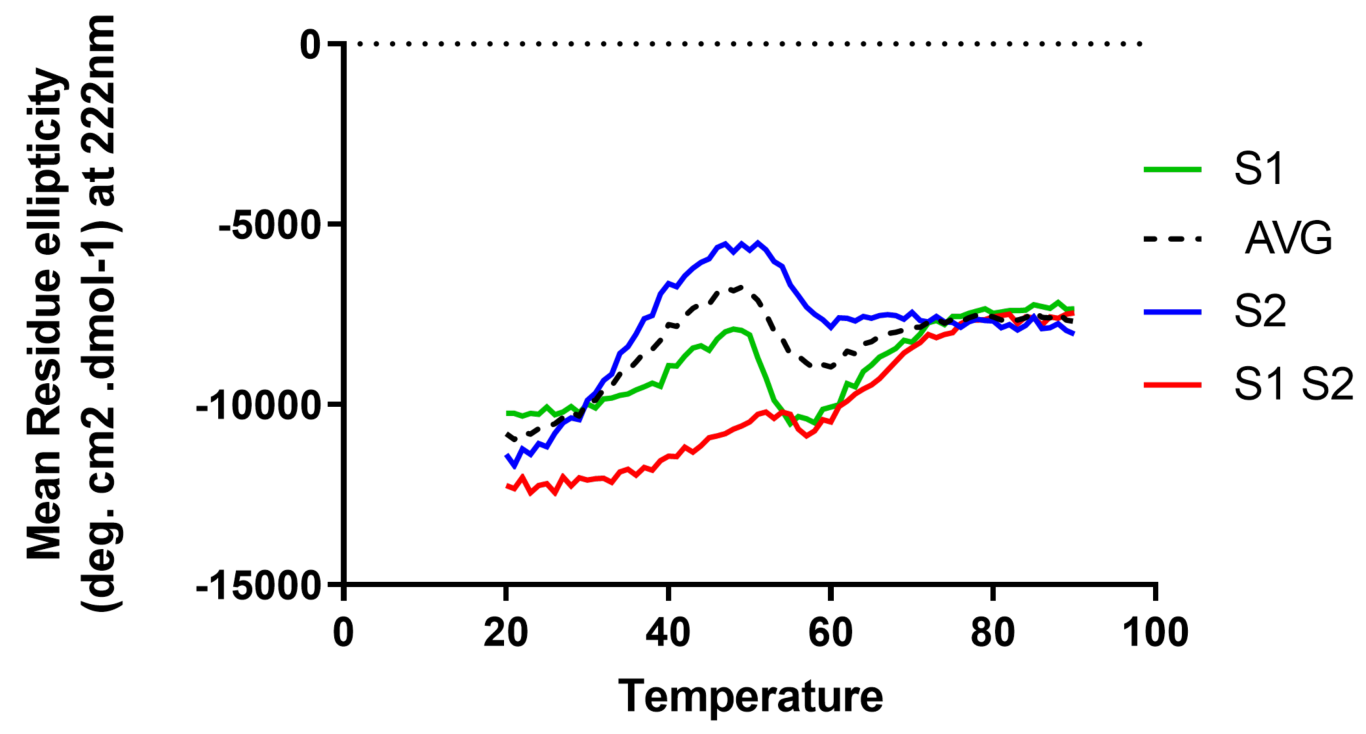

Supplementary Figure 5: Circular dichroism (CD) thermal denaturation profiles of S1, S2 and S1/S2 tracking mean residue ellipticity at $222 \mathrm{~nm}$. Top panel: S1, S2, S1/S2 and $\mathrm{S} 1+\mathrm{S} 2$ average (AVG) with the N-terminal charged peptide, bottom-panel: S1, S2, S1/S2 and $\mathrm{S} 1+\mathrm{S} 2$ average (AVG) without the charged peptide. The results show that a similar effect can be seen when mixing the two sub-units with and without the peptide. In both cases the profile resulting from the mixture is significantly from a theoretical average and exhibit a similar shape. The shape differences between S1 curves may attributed to experimental error. 


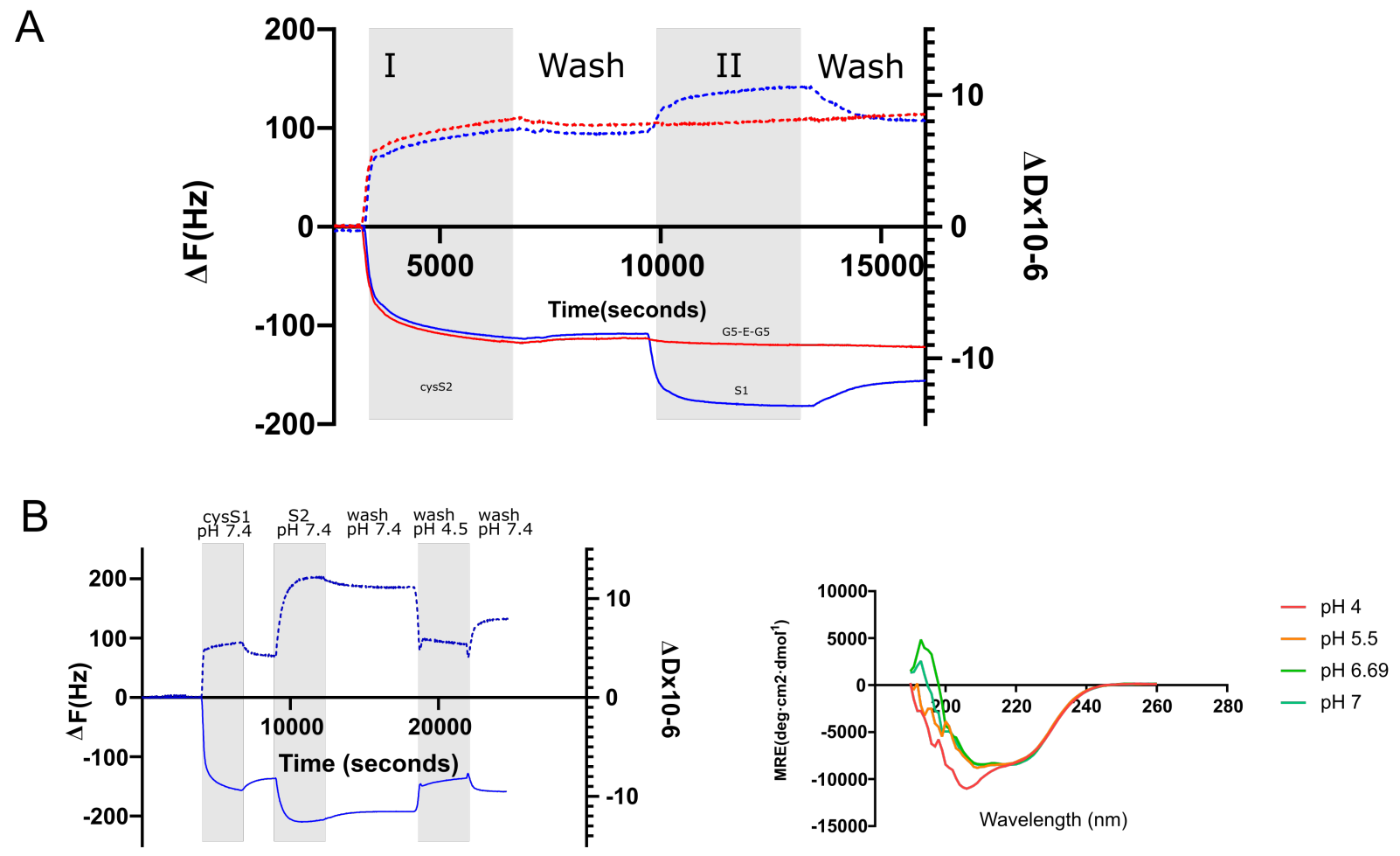

Supplementary Figure 6: QCM-D control experiment and $\mathrm{pH}$ dependent unfolding of a CysS2/S1 assembly (A) G5-E-G5 is washed over a layer of CysS2 (subsection II, red) with no binding, as expected as it lacks coiled-coil forming alpha helices. In a duplicate experiment, surface immobilized CysS2 is shown to bind S1 (blue), showing that the protein is capable for specific coiled-coil formation and does not engage in non-specific interactions with G5-E-G5. (B) A QCM-D experiment of CysS1 immobilized and bound to S2 (left) and subsequently washed off following addition of $20 \mathrm{mM}$ phosphate solution at $\mathrm{pH} 4.5$, most likely corresponding to a change in structure (transition to random coil), as observed in CD spectra of S1/S2 mixture following $\mathrm{pH}$ titration (right). 


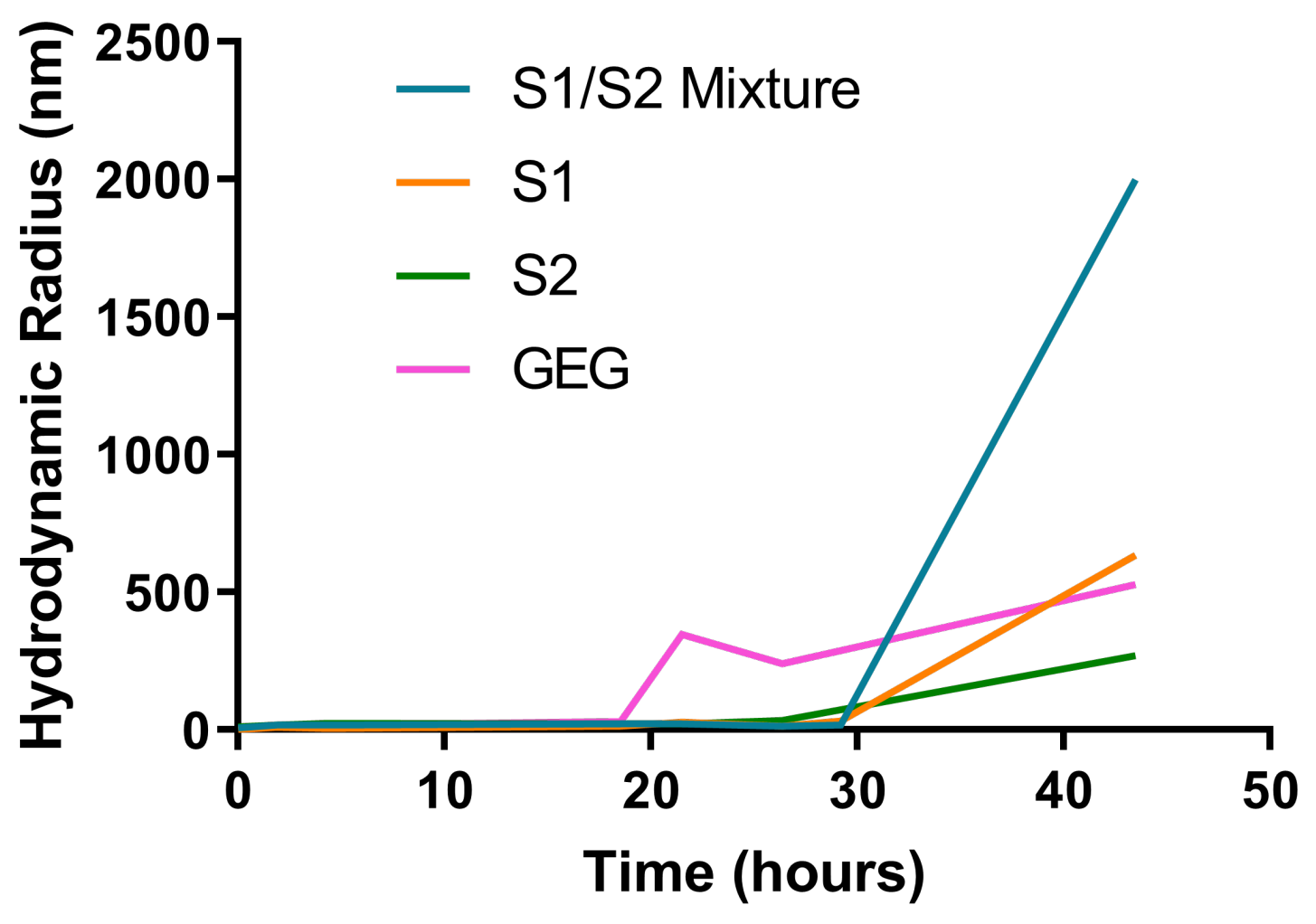

Supplementary Figure 7: A Dynamic Light Scattering experiment. $100 \mu \mathrm{g} \mathrm{ml}^{-1}$ protein incubations at room temperature. It can be seen that large objects become detectable starting from the 30-hour time point. This experiment was used to inform, when to start AFM visualization in liquid. G5-E-G5 most likely starts aggregating nonspecifically (visualizing G5-E-G5 in liquid AFM shows spherical aggregates at 30-hour time point). 


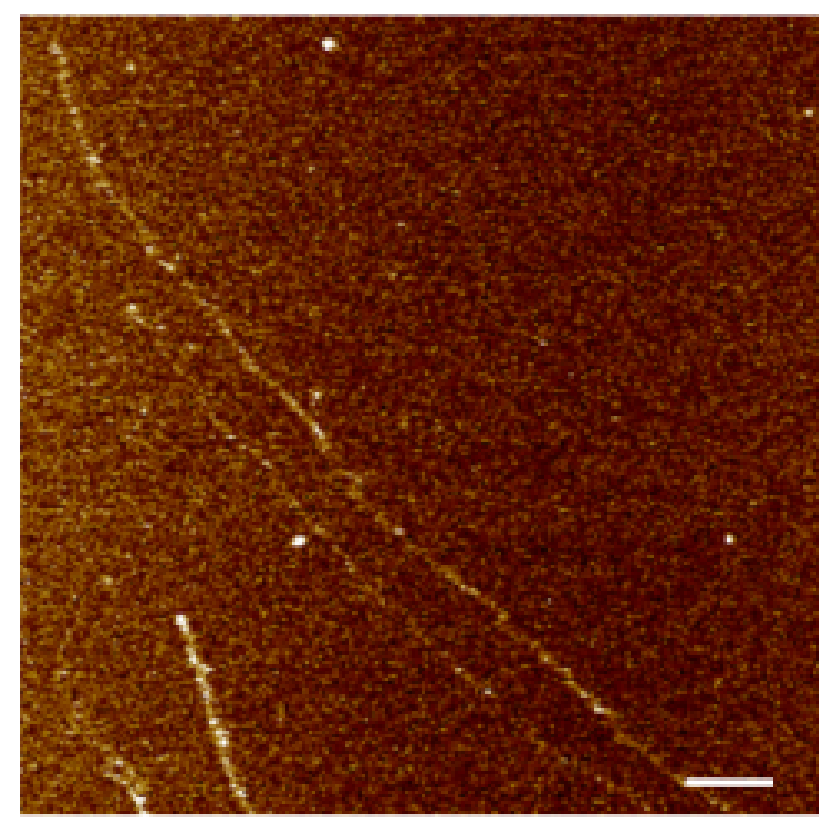

S1/S2

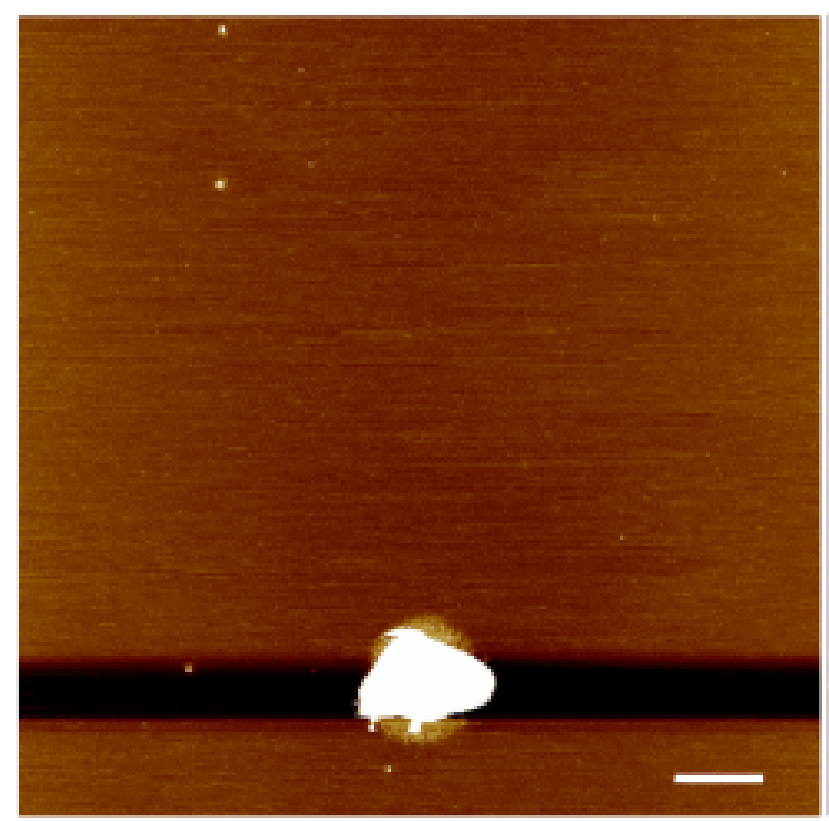

\section{G5-E-G5}

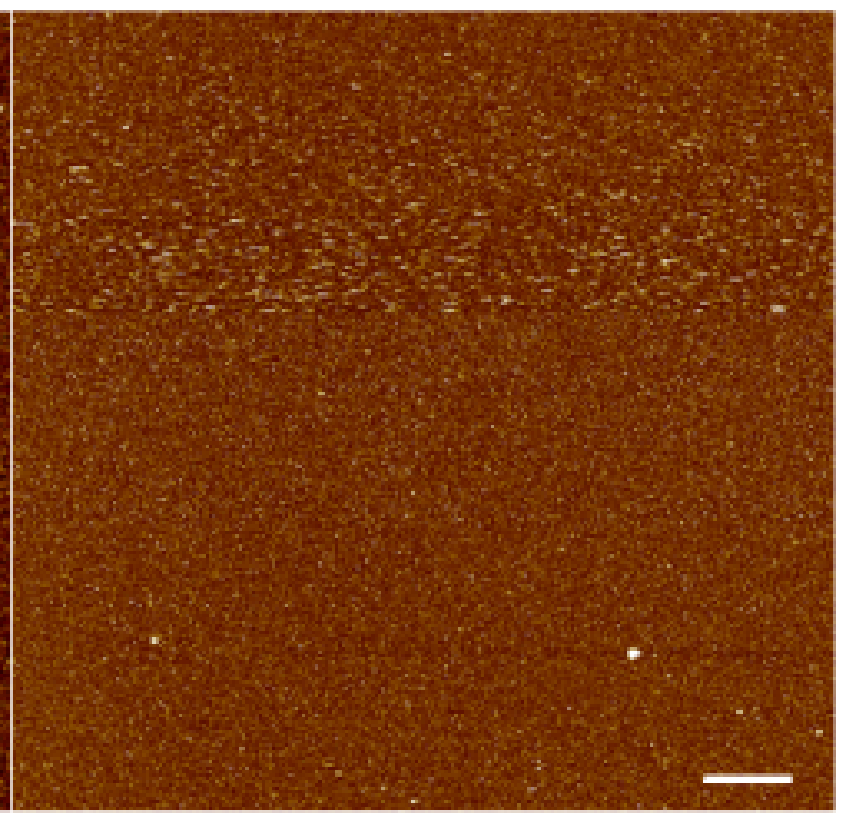

S1

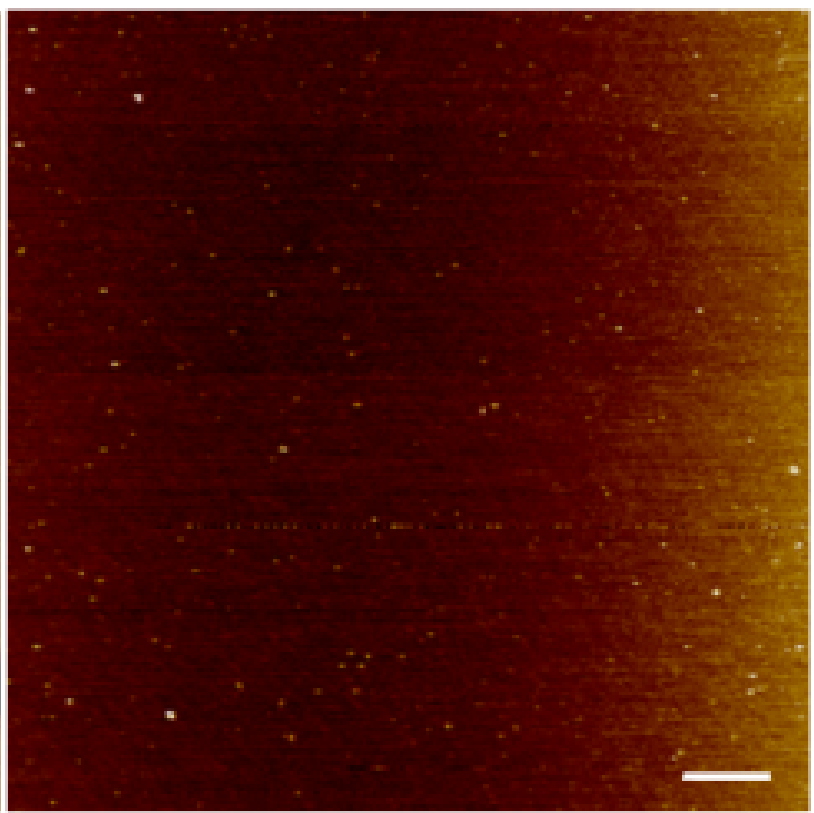

S2

Supplementary Figure 8: AFM liquid control experiment. Samples were prepared by incubating the proteins for 35 hours in room temperature at the concentration of $100 \mu \mathrm{g} \mathrm{ml}^{-1}$ then diluted to $1 \mu \mathrm{g} \mathrm{ml}^{-1}$ and applied to a poly-ornithine coated surface for 2 hours before gently washing with ultrapure water as in Figure 6 (in the main manuscript). Multiple scans of surfaces incubated with individual subunits S1 or S2 showed no large assemblies, implying that the assembly requires both subunits to be present. Visualized G5-E-G5 without coiled-coils shows spherical aggregates. Scale bars represent $500 \mathrm{~nm}$. 